\title{
Pathogens can slow down or reverse invasion fronts of their hosts
}

\author{
Frank M. Hilker ${ }^{1,2, *}$, Mark A. Lewis ${ }^{3}$, Hiromi Seno ${ }^{2}$, Michel Langlais ${ }^{4} \&$ \\ Horst Malchow ${ }^{1}$ \\ ${ }^{1}$ Institute of Environmental Systems Research, Department of Mathematics and Computer Science, University \\ of Osnabrück, Artilleriestr. 34, 49069 Osnabrück, Germany; ${ }^{2}$ Department of Mathematical and Life Sciences, \\ Graduate School of Science, Hiroshima University, Kagamiyama 1-3-1, Higashi-hiroshima 739-8526, Japan; \\ ${ }^{3}$ Department of Mathematical and Statistical Sciences, Department of Biological Sciences, University of \\ Alberta, Edmonton T6G 2G1, Canada; ${ }^{4} U M R$ CNRS 5466, Mathématiques Appliquées de Bordeaux, \\ Université Victor Segalen Bordeaux 2, 33076 Bordeaux Cedex, France; *Author for correspondence \\ (e-mail: fhilker@uos.de; fax: +49-541-969-2599)
}

Received 16 November 2004; accepted in revised form 22 February 2005

Key words: Allee effect, epidemiology, invasion, pathogen, reversal, SI model, slow-down, travelling wave

\begin{abstract}
Infectious diseases are often regarded as possible explanations for the sudden collapse of biological invasions. This phenomenon is characterized by a host species, which firstly can successfully establish in a nonnative habitat, but then spontaneously disappears again. This study proposes a reaction-diffusion model consisting of a simple SI disease with vital dynamics of Allee effect type. By way of travelling wave analysis, conditions are derived under which the invasion of the host population is slowed down, stopped or reversed as a consequence of a subsequently introduced disease. Hence, pathogens can dramatically control the rate of spread of invasive species.
\end{abstract}

\section{Introduction}

Biological invasions are regarded as one of the most severe ecological problems, being responsible for the extinction of indigenous species, sustainable disturbance of ecosystems and economic damage. Consequently, there is increasing need to control and manage invasions. This requires an understanding of the mechanisms underlying the invasion process. Recently, many factors have been identified that affect the speed and the pattern of the spread of an introduced species, either empirically and/or theoretically, such as stochasticity (Lewis 1997, 2000; Lewis and Pacala 2000; Malchow et al. 2004), resource availability, spatial heterogeneity (Murray 2003), environmental borders (Keitt et al. 2001), predation (Fagan and
Bishop 2000; Vinogradov et al. 2000; Owen and Lewis 2001; Petrovskii et al. 2002a, b), competition (Okubo et al. 1989; Hart and Garder 1997), evolutionary changes (Lambrinos 2004), largescale phenomena such as weather conditions or (long-range) dispersal/transport effects (Hengeveld 1989; Williamson 1996; Shigesada and Kawasaki 1997; Clark et al. 2001). For recent reviews see Fagan et al. (2002), Case et al. (2005), Hastings et al. (2005), Holt et al. (2005) and Petrovskii et al. (2005).

Substantial populations of introduced alien species are reported to establish successfully, but then to collapse spontaneously (Simberloff and Gibbons 2004). This phenomenon is also referred to as population crash. Pathogens are often suggested as an explanation, but in most cases there 
are neither documented empirical data nor identified pathogens.

Detailed theoretical studies of the influence of infectious diseases on the spread of species are still missing, but see Petrovskii et al. (2005), though there seems to be strong empirical evidence that pathogens and parasites play a major role in invasion biology, cf. Keane and Crawley (2002), Torchin et al. (2002), Clay (2003), Tompkins et al. (2003), Anderson et al. (2004), Lee and Klasing (2004), Prenter et al. (2004) and references therein.

The goal of this paper is to find conditions under which a subsequently released disease can be responsible for the decline, block or slowdown of established invasive species. Therefore, a two-component reaction-diffusion model describing the transmission of the pathogen and the spread of the host population is introduced in the next section. The host population exhibits a strong Allee effect (Dennis 1989; Courchamp et al. 1999; Stephens and Sutherland 1999; Stephens et al. 1999). The peculiar role of the Allee effect in biological invasions has been increasingly acknowledged in recent studies (Lewis and Kareiva 1993; Kot et al. 1996; Veit and Lewis 1996; Grevstad 1999; Takasu et al. 2000; Owen and Lewis 2001; Wang and Kot 2001; Garrett and Bowden 2002; Petrovskii et al. 2002a, b; Shigesada and Kawasaki 2002; Wang et al. 2002; Liebhold and Bascompte 2003; Cappuccino 2004; Davis et al. 2004; Drake 2004; Leung et al. 2004; Petrovskii et al. 2005).

Reaction-diffusion models are essential and analytically tractable tools for understanding invasion dynamics (Shigesada and Kawasaki 1997; Okubo and Levin 2001; Murray 2003). This study is based on travelling wave analysis (e.g. McKean 1970; Aronson and Weinberger 1975; Hadeler and Rothe 1975; Fife and McLeod 1977), in order to determine the front speeds which correspond to the asymptotic rate of spread. This approach can be traced back to the seminal works of Luther (1906), Fisher (1937), Kolmogorov et al. (1937) and Skellam (1951).

The outline of this paper is as follows. First, the model is described in the 'Mathematical model' section. Examples of different patterns of spread are then given by way of numerical simulations, including both the slow-down of invasion and the population decline to ultimate extinction. The travelling wave analysis in the fourth section reveals the conditions for the different behaviour. Finally, these results are discussed and related to similar work and a more general view of invasion patterns.

\section{Mathematical model}

We consider a spatiotemporal model for the spread of an invading species and its interplay with an introduced infectious disease. Therefore, the total population density $P=P(t, \boldsymbol{x}) \geq 0$ at time $t$ and spatial location $\boldsymbol{x}$ is split into a susceptible $S=S(t, \boldsymbol{x}) \geq 0$ and an infected part $I=I(t, \boldsymbol{x}) \geq 0$ with $P=S+I$. The epidemiological structure of the considered infectious disease is illustrated by the transfer diagram in Figure 1. Let $g(P)$ be the per-capita growth rate. We assume that the host population is subject to a strong Allee effect, say

$$
g(P)=a\left(K_{+}-P\right)\left(P-K_{-}\right),
$$

where $K_{+}>0$ is the carrying capacity and $K_{-}$, $0<K_{-}<K_{+}$, is the minimum viable population density, below which the disease-free population goes extinct due to the Allee effect. The parameter $a>0$ regulates the maximum growth rate. There is no recovery from the disease: Once a susceptible individual is exposed, it remains a lifelong carrier of the pathogen. Transmission between susceptibles and infected is via the standard incidence, also called proportionate mixing or frequency-dependent transmission, cf. Nold (1980), Hethcote (2000) and McCallum et al. (2001). Infected individuals suffer an additional disease-related mortality $\alpha>0$, also called the virulence. There is no vertical transmission, i.e.,

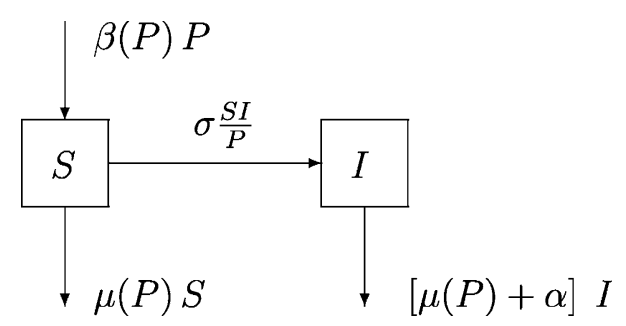

Figure 1. Transfer diagram of the infectious disease. More explanations can be found in the text. 
infected do not transmit the disease to their offspring. Thus, infected reproduce into the susceptible class, and the per-capita net growth rate is split into

$$
g(P)=\beta(P)-\mu(P)
$$

with $\beta(P) \geq 0$ being the fertility function and $\mu(P) \geq 0$ being the mortality function. We shall consider the following forms of $\beta(P)$ and $\mu(P)$, for which (1) holds:

$$
\begin{aligned}
& \beta(P)=a\left(-P^{2}+\left[K_{+}+K_{-}+e\right] P+c\right), \\
& \mu(P)=a\left(e P+K_{+} K_{-}+c\right),
\end{aligned}
$$

with parameters $c \geq 0$ and $e \geq 0$. The fertility function is quadratic and concave, i.e., $\beta(P)$ is increasing with $P$ for low densities, reaching a maximum value at some intermediate density and decreasing for high densities. This may be due to biological reasons inducing the Allee effect, see the references in the introduction. The mortality function is assumed to increase linearly with the population density as it is usually assumed in logistic growth models. Note that with increasing values of the parameter $e$, the decreasing branch of the fertility function becomes less prominent in the range $P \in\left[0, K_{+}\right]$. In the mortality function, the effect of density dependence increases with $e$. The parameter $c$ controls both vital functions in a density-independent way. Strictly speaking, Equations (2-3) make sense as long as the fertility function remains nonnegative; this holds true at least for $0 \leq P \leq K_{+}+K_{-}+e$, which is sufficient for this model. Together with diffusion as spatial propagation mechanism, we obtain a system of two partial differential equations (PDEs)

$$
\begin{aligned}
& \frac{\partial S}{\partial t}=-\sigma \frac{S I}{P}+\beta(P) P-\mu(P) S+D_{S} \Delta S, \\
& \frac{\partial I}{\partial t}=\sigma \frac{S I}{P}-\mu(P) I-\alpha I+D_{I} \Delta I
\end{aligned}
$$

where $\Delta$ is the Laplacian and $\sigma>0$ the transmission coefficient. This model has been proposed and investigated by Hilker et al. (submitted) for equal diffusion coefficients $D=D_{S}=D_{I}$, because the disease is assumed not to affect the spreading behaviour. The same assumption will be used in this paper.
In their numerical simulations, they found that a spreading population can go extinct when the disease is introduced supplementarily. This is a spatial phenomenon, because in the spatially homogeneous case both the host population and the disease coexist. This paper is concerned with an analytical explanation of this effect. Therefore, we restrict ourselves to one-dimensional space, i.e. $\boldsymbol{x}=x$, and introduce the dimensionless quantities

$$
\begin{array}{lll}
\tilde{S}=\frac{S}{K_{+}}, & \tilde{t}=a K_{+}^{2} t, & \tilde{x}=K_{+} \sqrt{\frac{a}{D}} x, \\
\tilde{I}=\frac{I}{K_{+}}, & \tilde{u}=\frac{K_{-}}{K_{+}} \in(0,1), & \tilde{\alpha}=\frac{\alpha}{a K_{+}^{2}}>0, \\
\tilde{\sigma}=\frac{\sigma}{a K_{+}^{2}}>0, & \tilde{c}=\frac{c}{K_{+}^{2}} \geq 0, & \tilde{e}=\frac{e}{K_{+}} \geq 0 .
\end{array}
$$

Dropping the tildes for notational simplicity, we thus obtain for (4-5) the system

$$
\begin{aligned}
\frac{\partial S}{\partial t}= & -\sigma \frac{S I}{P}+[(1+u+e-P) P+c] P \\
& -(e P+u+c) S+\frac{\partial^{2} S}{\partial x^{2}} \\
\frac{\partial I}{\partial t}= & {\left[\sigma \frac{S}{P}-e P-u-c-\alpha\right] I+\frac{\partial^{2} I}{\partial x^{2}}, }
\end{aligned}
$$

where $P=S+I$ now denotes the dimensionless total population density. The number of parameters has been reduced from eight to five. System (6-7) is studied along with no-flux boundary conditions. Because we are interested in an invasion problem with a subsequently introduced disease in the wake of the invasion, we differentiate three regions of initial conditions. Without loss of generality, the right boundary is assumed to be still non-invaded. In the middle, there are only susceptibles. At the left boundary, the disease is assumed to have been introduced such that susceptibles and infected coexist:

$$
\begin{aligned}
& S(0, x)=S_{l}>0 \text { if } x<x_{S} \text { and } 0 \text { otherwise, } \\
& I(0, x)=I_{l}>0 \text { if } x<x_{I} \text { and } 0 \text { otherwise, }
\end{aligned}
$$

with $x_{S}>x_{I}>0$. There is a singularity in system (6-7) at the trivial solution. Introducing the prevalence $i=I / P \in[0,1]$, we reformulate (6-9) in $(P, i)$ space: 


$$
\begin{aligned}
& \frac{\partial P}{\partial t}=[(1-P)(P-u)-\alpha i] P+\frac{\partial^{2} P}{\partial x^{2}}, \\
& \frac{\partial i}{\partial t}=[\sigma-\alpha-c-(1+u+e-P) P-(\sigma-\alpha) i] i \\
& +\frac{2}{P} \frac{\partial P}{\partial x} \frac{\partial i}{\partial x}+\frac{\partial^{2} i}{\partial x^{2}}, \\
& P(0, x)= \begin{cases}S_{l}+I_{l} & \text { if } x<x_{I}, \\
S_{l} & \text { if } x_{S} \leq x<x_{I} \\
0 & \text { if } x \geq x_{S},\end{cases} \\
& i(0, x)= \begin{cases}I_{l} /\left(S_{l}+I_{l}\right) & \text { if } x<x_{I} \\
0 & \text { if } x_{I} \leq x<x_{S} \\
i_{r} \in[0,1] & \text { if } x \geq x_{S} .\end{cases}
\end{aligned}
$$

Note the convenient expressions in (10), but due to the transformation, (11) contains a term with the inverse of $P$ and the spatial derivatives of both $P$ and $i$. However, if we consider the spatially homogeneous version of (10-11), the singularity vanishes. The stationary states and their stabilities are summarized in Table 1. They have been found by Hilker et al. (submitted) for a generalized model with $\beta(P)$ being concave and $\mu(P)$ being nondecreasing and convex. There may be up to six equilibria. Periodic solutions are proven not to exist (Poincaré-Bendixson theory). The dynamics are bistable. If $\sigma-\alpha<c$, the disease cannot establish and the host population either goes extinct or approaches the carrying capacity, depending on the initial conditions.
Further increasing the disease-related parameter combination $\sigma-\alpha$, the trivial solution becomes unstable and the disease-induced extinction state $\left(0, i_{2}=1-\frac{c}{\sigma-\alpha}\right)$ emerges. This equilibrium corresponds to extinction as a consequence of infection (de Castro and Bolker 2005). While the host population tends to zero, the prevalence approaches $i_{2}>0$. Next, if $\sigma-\alpha>u+c+u e$, up to two coexistence states appear. The smaller one $\left(P_{3-}, i_{3-}\right)$ is always unstable, while the larger one $\left(P_{3+}, i_{3+}\right)$ changes stability with the disease-free carrying capacity state and is locally stable as soon as it exists. The nontrivial equilibria disappear if additionally

$$
\begin{aligned}
& e>\frac{\sigma}{\alpha}(1-u), \\
& c<\frac{(\sigma-\alpha)(u+\alpha)}{\alpha}-\frac{[\sigma(1+u)+\alpha e]^{2}}{4 \alpha \sigma} .
\end{aligned}
$$

In this case the dynamics are monostable with a globally stable disease-induced extinction state. The total population densities of the coexistence states are

$$
\begin{aligned}
& P_{3+, 3-}=\frac{1}{2}\left[1+u+\frac{e \alpha}{\sigma}\right. \\
& \left. \pm \sqrt{\left(1+u+\frac{e \alpha}{\sigma}\right)^{2}-\frac{4}{\sigma}[(\sigma-\alpha)(u+\alpha)-c \alpha]}\right]
\end{aligned}
$$

They can be found as the roots of the two nontrivial, quadratic nullclines

Table 1. Results of the stability analysis of the spatially homogeneous version of (10-11).

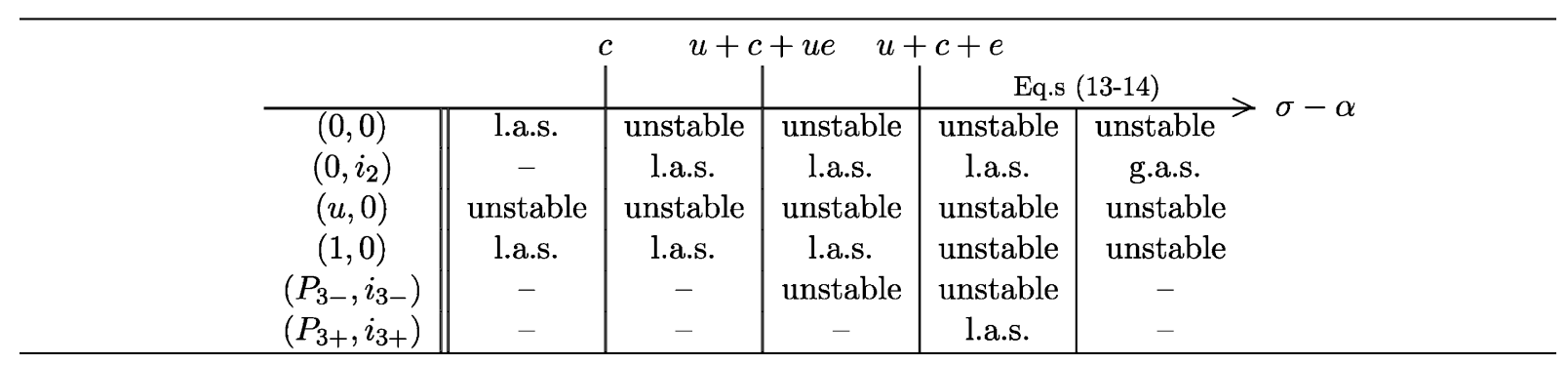

The left column contains the stationary states $\left(P^{*}, i^{*}\right)$. The other columns are divided according to the parameter regions along the ray for $\sigma-\alpha$ which are separated by the values in the top row. The most right column corresponds to the case that Equations (13-14) hold additionally. 'l.a.s.' stands for locally asymptotically stable, 'g.a.s.' for globally asymptotically stable, and '-' means that the stationary state does not exist or is not feasible. 


$$
\begin{aligned}
i(P) & =\frac{(1-P)(P-u)}{\alpha} \\
& =\frac{\sigma-\alpha-c-(1+u+e-P) P}{\sigma-\alpha},
\end{aligned}
$$

which are displayed, for example, in Figure 6a.

\section{Slow-down and reversal of invasion fronts: numerical observations}

When the invasion front of the host population is not influenced by the disease, for example if $i=0$, system (10-11) reduces to a single-species model in which growth is described by a strong Allee effect. In this case, the invasion takes place via a travelling frontal wave with the unique speed

$$
v=\frac{1}{\sqrt{2}}(1-2 u),
$$

cf. Lewis and Kareiva (1993). If $u<1 / 2$, the population front propagates in positive direction, while it runs backward otherwise.

In this paper we assume that the population initially propagates forward, say $0<u<1 / 2$, and still continues spreading when the disease is introduced in the wake of the invasion front of the host species, i.e.

- we restrict ourselves to the parameter region in which the coexistence state is stable, cf. Table 1 ,

- the initial conditions prevent mere extinction due to the Allee effect, say (i) at least $P_{l}>u$ and (ii) the spatially critical threshold length is exceeded (Hilker et al. submitted), i.e. $x_{S}$ is large enough.

This section is concerned with numerical simulations. The second summand on the right-hand side of (11) causes enormous numerical difficulties. Note that it can be interpreted as an advection term with velocity

$$
-\frac{2}{P} \frac{\partial P}{\partial x},
$$

i.e. the direction of the advection depends on the spatial gradient of $P$. The term does not play a role at the head and the wake of the fronts, because there at least one of the spatial derivatives vanishes. However, this term could become important if the transition layers of both fronts for $P$ and $i$ overlap. Therefore, we consider for the numerical simulations system (6-9). In order to numerically handle the singularity, a threshold $\delta$ is applied, below which the transmission terms are set to zero. Throughout this paper, it will be kept as small as $\delta=10^{-100}$. As will become evident below, the underlying reason is that the extinction state $S=I=0$ is stable. Experiences from numerical simulations elucidate that this extremely small choice of $\delta$ is necessary, in order to avoid artificial effects at the propagating wave front. Moreover, a sufficiently small time discretization is required.

Extensive numerical investigations indicate the emergence of travelling frontal waves. An example is given in Figure 2, where the numerical solutions are displayed in $P$ and $i$ state variables. At the head of the population front, the host species continues invasion in open space with speed $v_{1}$. The infection spreads into the susceptible population at carrying capacity with speed $v_{2}$ and settles towards the coexistence state (Figure 2a). If the prevalence front comes closer to the population front, there is a sudden boost of the prevalence in front of the population front (Figures 2b, c). Due to diffusion, there is a band of microscopic prevalence values moving ahead the actual prevalence front. Together with small total population densities, the system dynamics quickly tends to the disease-induced extinction state, because the trivial state is unstable. The travelling wave thus connects the coexistence state with the disease-induced extinction state, which has to establish from the trivial state. A too small choice of the threshold $\delta$ in the numerical scheme would affect an abrupt interruption of the rapid propagation of the disease-induced extinction state through the empty space (with speed $v_{3}$ ). Note that it is instructive to display the dynamics in $P$ and $i$, because otherwise the decisive distinction between the unstable trivial state $(0,0)$ and the stable disease-induced extinction state $\left(0, i_{2}\right)$ would be not as obvious.

The appearance of the prevalence "hump" in front of the front is a transient effect of small densities, which has also been referred to as the "atto-fox problem" (Mollison 1991). Since this is often regarded as artificial, note that the effect could be damped by simply applying an appropriate threshold, below which densities are set to zero, cf. Gurney et al. (1998), Gurney and Nisbet 

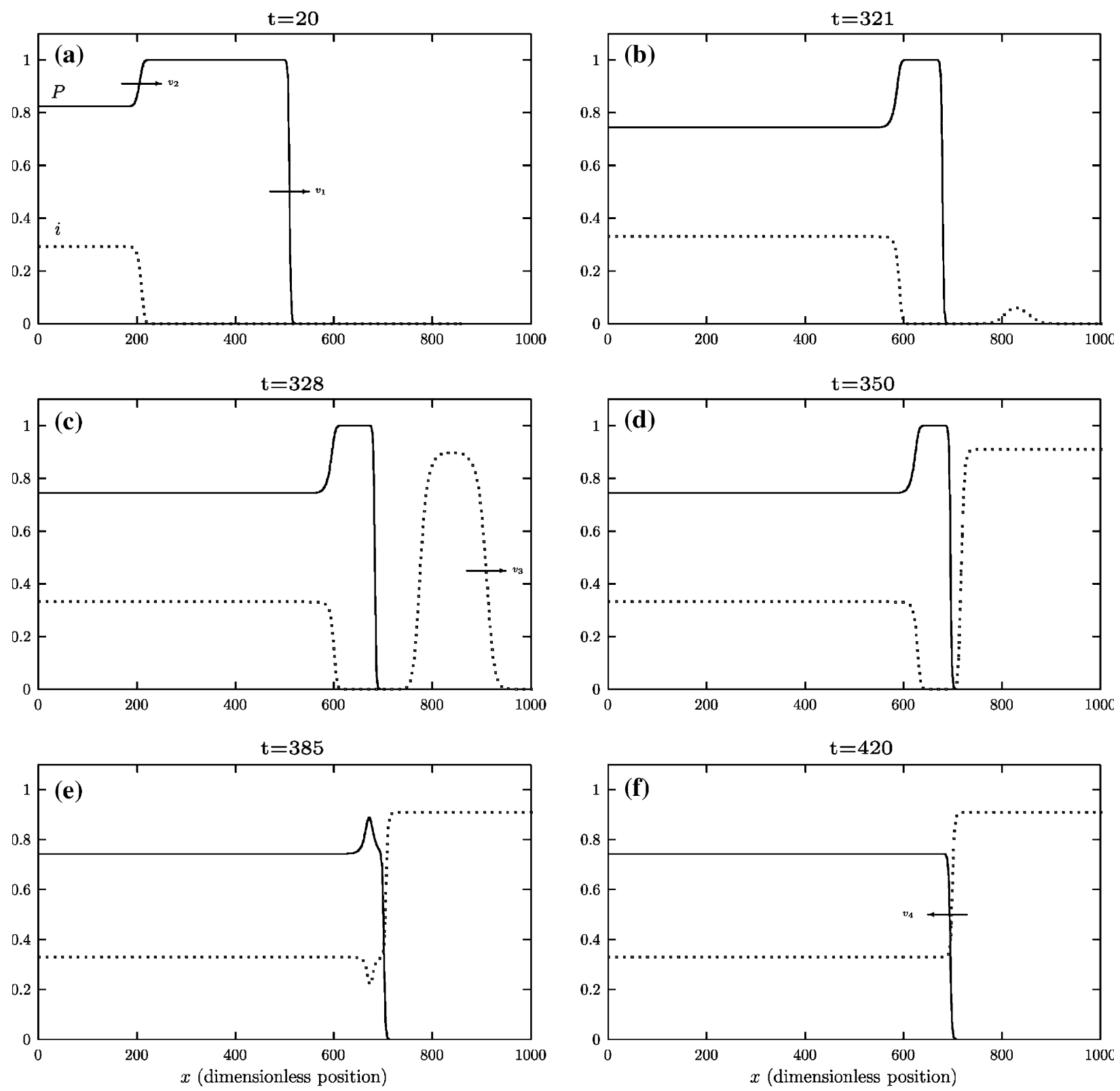

Figure 2. Reversal of invasion fronts, when the introduced disease catches up the front of the host population. The solid line represents $P$, the dotted line $i$. Solutions are obtained, as throughout this paper, by integrating (6-9) with the Runge-Kutta scheme of fourth order for local interactions, with the explicit Euler scheme for diffusion and applying a threshold as described in the text due to the singularity at the trivial state. Other parameter values: $\alpha=0.5, \sigma=1.6, c=0.1, e=0.5, u=0.1, S_{l}=1, I_{l}=0.001, x_{S}=500$, $x_{I}=200$.

(1998) and Cruickshank et al. (1999). The results, however, would qualitatively remain the same, namely the disease-induced extinction state propagates to the right-hand side, while the coexistence state moves with speed $v_{4}$. In the example shown, the population front becomes recessive (Figures $2 \mathrm{~d}-\mathrm{f}$ ), and the population ultimately goes extinct. For other parameter values, e.g. a smaller value of the virulence, the population front remains invasive, but with a slower wave 


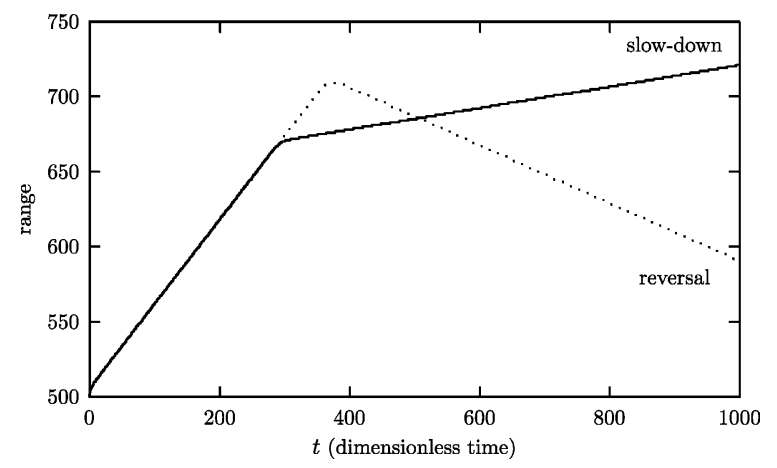

Figure 3. Range expansion of the total population for $\alpha=0.3$ (slow-down) and $\alpha=0.5$ (reversal). The range is the (dimensionless) distance which is occupied by the species with densities above some threshold, here set to 0.01 . The slopes of the displayed lines correspond to the wave speeds. Other parameters as in Figure 2.

speed, i.e. $v_{4}<v_{1}$. The reversal and slow-down of the invasion fronts are illustrated in the range expansion diagram in Figure 3.

The next section is concerned with finding analytical conditions for either the slow-down or the reversal. This is achieved by deriving approximations for the wave speed $v_{4}$.

\section{Travelling wave analysis}

Although there are some results proving the existence of travelling wave solutions to systems similar to (6-9), e.g. Dunbar (1983, 1984, 1986), Hosono (1989), Huang et al. (2003) and Li et al. (submitted), we know of no proof of travelling wave solutions for this specific system. For the case that one species (predator) spreads into the range of another spreading species (prey), simulations by Sherratt et al. $(1995,1997)$ and Petrovskii and Malchow (1999, 2001) show that spatiotemporal dynamics can result in irregular oscillations - provided that the local dynamics fulfill some special conditions. Since these requirements differ from the properties of our model, we do not expect such a behaviour. As a result from the numerical simulations in the previous section, we consider travelling wave solutions to $(10-12)$ of the form $\tilde{P}(z)=P(t, x)$ and $\tilde{i}(z)=i(t, x)$ with $z=x-v t$ and wave speed $v$. Omitting the tildes for notational simplicity and substituting, we get the following system of ordinary differential equations of second order

$$
\begin{aligned}
0= & P^{\prime \prime}+v P^{\prime}+[(1-P)(P-u)-\alpha i] P, \\
0= & i^{\prime \prime}+\left(v+\frac{2}{P} P^{\prime}\right) i^{\prime} \\
& +[\sigma-\alpha-(1+u+e-P) P-c \\
& -(\sigma-\alpha) i] i
\end{aligned}
$$

where the prime denotes differentiation with respect to $z$. Focusing on the situation discussed last in the previous section, cf. Figure 2f, we specify the boundary conditions as

$$
\begin{gathered}
P(-\infty)=P_{3+}, \quad P(\infty)=0, \\
i(-\infty)=i_{3+}, \quad i(\infty)=i_{2} .
\end{gathered}
$$

Our main interest is in the direction of the travelling wave, say whether it moves to the left or to the right. Because of this we consider Equation (18) for the total population density. By introducing $Q=P^{\prime}$, this single equation of second order can be reduced to a system of two equations of first order, namely

$$
\begin{aligned}
& P^{\prime}=Q \\
& Q^{\prime}=-v Q-[(1-P)(P-u)-\alpha i] P,
\end{aligned}
$$

with boundary conditions

$$
\begin{array}{ll}
P(-\infty)=P_{3+}, & P(\infty)=0, \\
Q(-\infty)=0, & Q(\infty)=0 .
\end{array}
$$

Note that (21) depends on the prevalence $i$, which induces mathematical difficulties. In the subsection 'Exact wave speed solutions for fast dynamics', we will determine exact wave speeds for cases, in which we can make use of different time scale dynamics. This enables us to approximate the prevalence. In the subsection 'Approximations for small $\sigma$, we show that we can also find good approximations for other cases.

\section{Exact wave speed solutions for fast dynamics}

Our model depends on five parameters, cf. (1819). However, $u$ is restricted to be within the interval $(0,0.5)$, cf. section 'Slow-down and reversal of invasion fronts: numerical observations'. Furthermore, the virulence $\alpha$ cannot be very large, because otherwise there are too many deaths due to the 
disease, and vital dynamics would become irrelevant. Thus, there remain the three parameters $\sigma, e$ and $c$. In this subsection, we will investigate the cases in which the transmission coefficient $\sigma$ becomes very large. This situation may arise when the disease is transmitted very fast. Moreover, the combinations of additionally large growth parameters $e$ and/or $c$ are also taken into account. In all these cases, a unique wave speed can be approximated analytically. The case that $\sigma$ is small will be considered in the next subsection.

Firstly, we assume that both the disease transmission as well as the vital dynamics (births and deaths) occur on a fast time scale, i.e., we replace $e, c$ and $\sigma$ by $e / \varepsilon, c / \varepsilon$ and $\sigma / \varepsilon$, respectively, and then let $\varepsilon \rightarrow 0$. Consider Equation (11) for $i$. Substituting $e, c$ and $\sigma$ as indicated and multiplying with $\varepsilon$ yield

$$
\begin{aligned}
\varepsilon \frac{\partial i}{\partial t}= & {[\sigma-\varepsilon \alpha-c-(\varepsilon+\varepsilon u+e-\varepsilon P) P-(\sigma-\varepsilon \alpha) i] i } \\
& +\varepsilon \frac{2}{P} \frac{\partial P}{\partial x} \frac{\partial i}{\partial x}+\varepsilon \frac{\partial^{2} i}{\partial x^{2}} .
\end{aligned}
$$

With $\varepsilon \rightarrow 0$, this reduces to

$$
0=[\sigma-c-e P-\sigma i] i,
$$

and thus $i=0$, which corresponds to the diseasefree states, or

$$
i=1-\frac{c}{\sigma}-\frac{e}{\sigma} P
$$

for cases in which the infection can establish. The total population density of the stable coexistence state reduces to

$$
\begin{aligned}
P_{3+}=\frac{1}{2}[1 & +u+\frac{e \alpha}{\sigma} \\
& \left.+\sqrt{\left(1+u+\frac{e \alpha}{\sigma}\right)^{2}-4\left(u+\alpha-\frac{c \alpha}{\sigma}\right)}\right] .
\end{aligned}
$$

Hence, the prevalence can be described by the linearly decreasing straight line (22), cf. Figure 4a. Incorporating this in (20-21) yields

$$
\begin{aligned}
& P^{\prime}=Q \\
& Q^{\prime}=-v Q-\left[(1-P)(P-u)-\alpha\left(1-\frac{c}{\sigma}-\frac{e}{\sigma} P\right)\right] P .
\end{aligned}
$$

We are looking for a heteroclinic connection between $(P, Q)=\left(P_{3+}, 0\right)$ and $(P, Q)=(0,0)$. Therefore, following Lewis and Kareiva (1993) and Murray (2002), we make the ansatz

$$
P^{\prime}=Q=A P\left(P_{3+}-P\right) .
$$

Then

$$
P^{\prime \prime}=A P^{\prime}\left(P_{3+}-2 P\right)=A^{2} P\left(P_{3+}-P\right)\left(P_{3+}-2 P\right),
$$

and substituting these expressions together with (22) in (18) yields

$$
\begin{aligned}
0= & {[\underbrace{\left(2 A^{2}-1\right)}_{c_{2}} P^{2}+\underbrace{\left(1+u+\frac{\alpha e}{\sigma}-v A-3 A^{2} P_{3+}\right)}_{c_{1}} P} \\
& \underbrace{-u-\alpha+\frac{c \alpha}{\sigma}+v A P_{3+}+A^{2} P_{3+}^{2}}_{c_{0}}] P .
\end{aligned}
$$

This equation must hold for all $P \in\left[0, P_{3+}\right]$. For $P=0$ it obviously does, and for all other values of $P$ we require that $c_{2}=c_{1}=c_{0}=0$. From $c_{2}=0$ we obtain a condition for $A$, namely

$$
A= \pm \frac{1}{\sqrt{2}}
$$

and from the remaining two conditions $c_{1}=c_{0}=0$ we obtain equations for the wave speed $v$. They must be equal, which they are, indeed, for the values of $A$ as above and $P_{3+}$ as in (23).

Because we are looking for a connection from $\left(P, P^{\prime}\right)=\left(P_{3+}, 0\right)$ to $\left(P, P^{\prime}\right)=(0,0), \quad P$ must decrease, which means that $P^{\prime} \leq 0$ and that the trajectory is situated in the fourth quadrant. Hence, choosing $A=-1 / \sqrt{2}$, we obtain the unique wave speed

$$
\begin{aligned}
v= & \frac{\sqrt{2}}{4}\left[-1-u-\frac{e \alpha}{\sigma}\right. \\
& \left.+3 \sqrt{\left(1+u+\frac{e \alpha}{\sigma}\right)^{2}-4\left(u+\alpha-\frac{c \alpha}{\sigma}\right)}\right]
\end{aligned}
$$

Figure 5a shows (24) in comparison with the numerically obtained values, demonstrating that the speeds match very well.

Next, we can repeat this procedure analogously for all other cases of fast dynamics, 

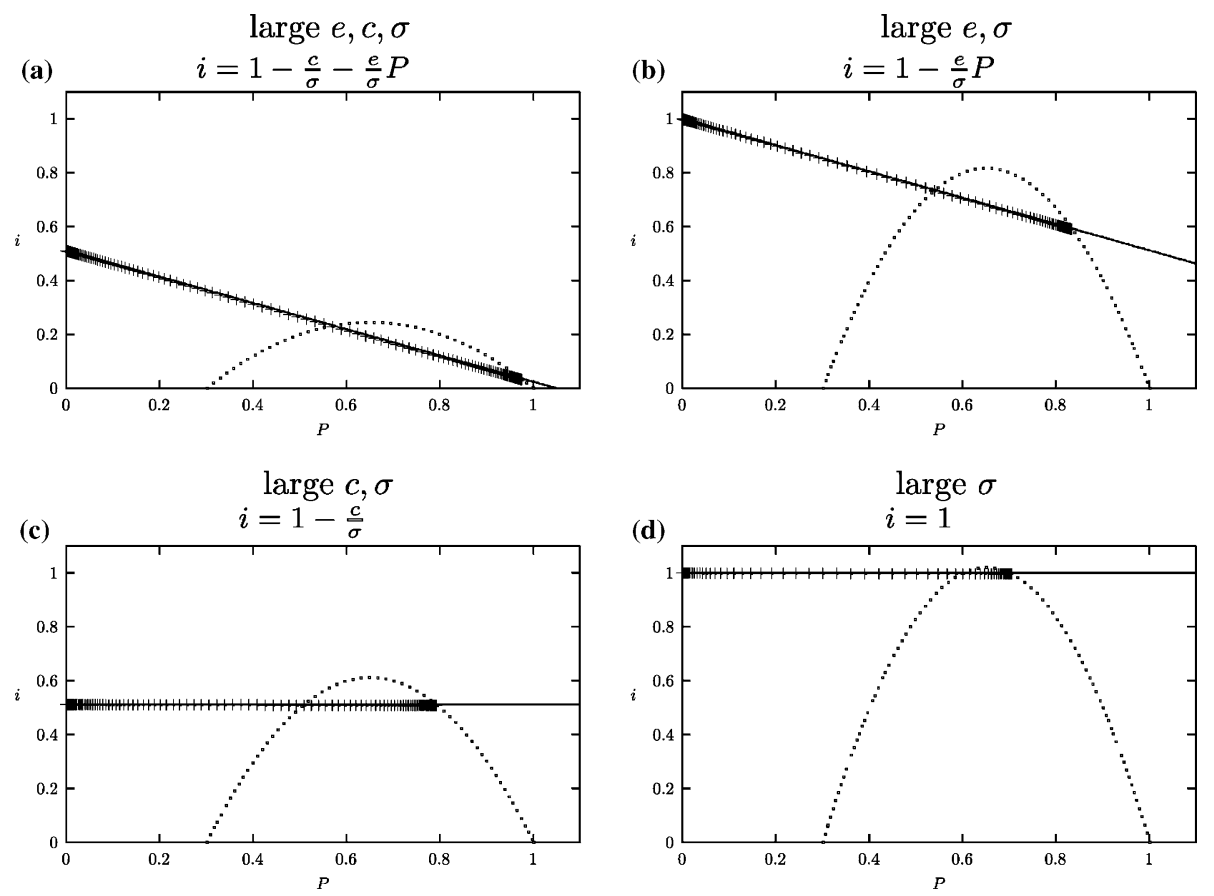

Figure 4. Solutions of the PDE system (6-9), restricted to the asymptotic propagation of the travelling fronts, displayed in $(P, i)$ phase plane with cross points, for different cases of fast dynamics. The solid lines are the prevalence approximations as given in the respective panel. The dotted lines are the nullclines of the total population, cf. the left part of (16). Parameter values: (a) $e=c=100.0, \sigma=205.0, \alpha=0.5$, (b) $e=100.0, c=0.1, \sigma=205.0, \alpha=0.15$, (c) $e=100.0, c=0.1, \sigma=205.0, \alpha=0.2,(\mathrm{~d}) e=c=0.1$, $\sigma=205.0, \alpha=0.12$. All other values have been kept as $u=0.3, S_{l}=S_{3+}, I_{l}=I_{3+}, x_{S}=500, x_{I}=200$.

(a)
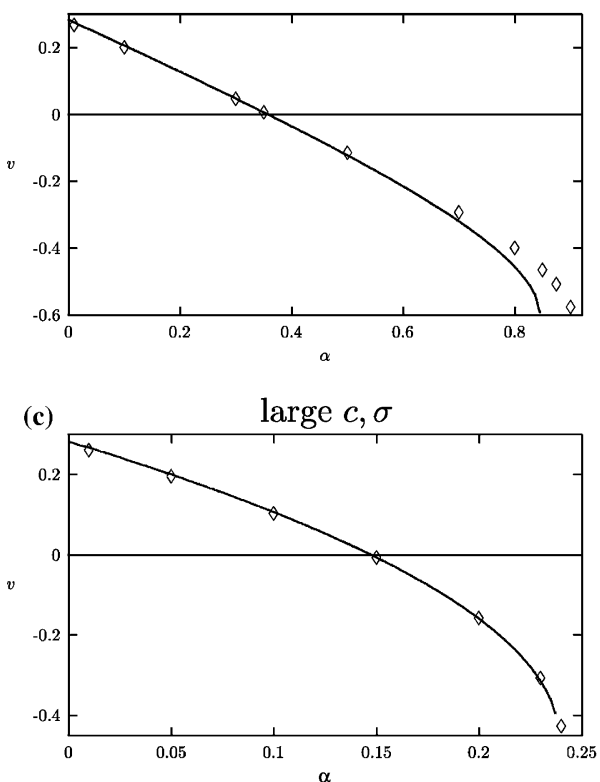

(b)

large $e, \sigma$

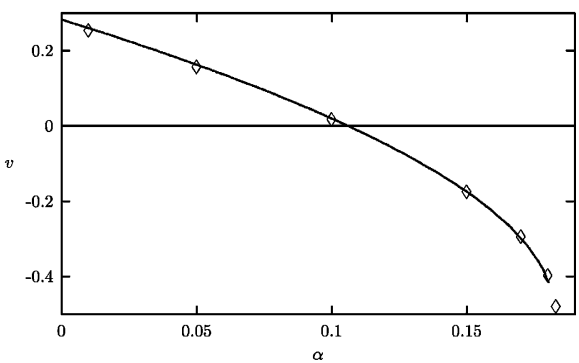

(d)

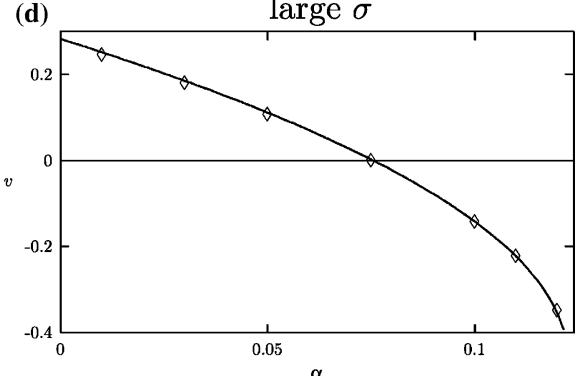

Figure 5. Analytically derived wave speeds (24-27) for the different combinations of fast transmission and vital dynamics, cf. Figure 4 (same parameter values). The points are numerically obtained results. 
obtaining approximations for the prevalence as given and plotted in Figures $4 \mathrm{~b}-\mathrm{d}$. Note that a large $c$ corresponds to the situation in which both the fertility as well as the mortality function occur on a fast time scale. For a large $e$, the effect of density dependence is increased. Hence, the dynamics become fast for high densities. The respective approximations of $i$ are in all cases straight lines. If $e$ is large, then they depend on $P$ (Figures $4 \mathrm{a}-\mathrm{b}$ ). Otherwise, $i$ can be approximated by a constant. For large values of $c$, this constant is below unity (Figure 4c), otherwise it is exactly unity (Figure 4d). The corresponding wave speeds are

$$
\begin{aligned}
v= & \frac{\sqrt{2}}{4}\left[-1-u-\frac{e \alpha}{\sigma}\right. \\
& \left.+3 \sqrt{\left(1+u+\frac{e \alpha}{\sigma}\right)^{2}-4(u+\alpha)}\right] \quad(\text { large } e, \sigma),
\end{aligned}
$$

$$
\begin{aligned}
v= & \frac{\sqrt{2}}{4}[-1-u \\
& \left.+3 \sqrt{(1+u)^{2}-4\left(u+\alpha-\frac{c \alpha}{\sigma}\right)}\right] \quad(\text { large } c, \sigma),
\end{aligned}
$$

$$
v=\frac{\sqrt{2}}{4}\left[-1-u+3 \sqrt{(1-u)^{2}-4 \alpha}\right]
$$

The plots in Figures $5 \mathrm{~b}-\mathrm{d}$ underline the good approximation of the wave speeds obtained in numerical simulations. The analytic solutions reveal that the waves exist, i.e. $v$ is a real number, as soon as the stable coexistence states exist in the corresponding situations, cf. for instance (23). Note that $v$ can become negative. In this case, there is a reversal of the invasion front. A zero-velocity corresponds to the stop of the invasion. Though such a stationary front is superimposed by noisy fluctuations in nature and thus unlikely to be observed, this can be an explanation for at least transient species borders in spatially homogeneous environments, cf. Holt et al. (2005) and references therein. In the case that exclusively $\sigma$ is large, the speed only depends on $u$ and $\alpha$. In the other cases, the speed additionally depends on $e, c$ and $\sigma$. To be more precise, just the ratios of $e / \sigma$ and $c / \sigma$ influence the rate of spread. Note that in all the cases of fast dynamics, the prevalence is approximated by its nullcline of the non-spatial model. This is due to the fact that the disease transmission occurs on the fast time scale.

\section{Approximations for small $\sigma$}

If $\sigma$ is small, which corresponds to a slow transmission of the disease, while the vital dynamics occur on a fast time scale, i.e. one or both of the parameters $e$ and $c$ are large, then the disease cannot establish. This means that there is no coexistence state, cf. the parameter regions in Table 1. In the last remaining situation neither the disease transmission nor the vital dynamics occur on a fast time scale. In this case, the prevalence cannot be approximated as before. However, experiences from numerical simulations indicate that, nonetheless, an estimate of the unique wave speed can be obtained.
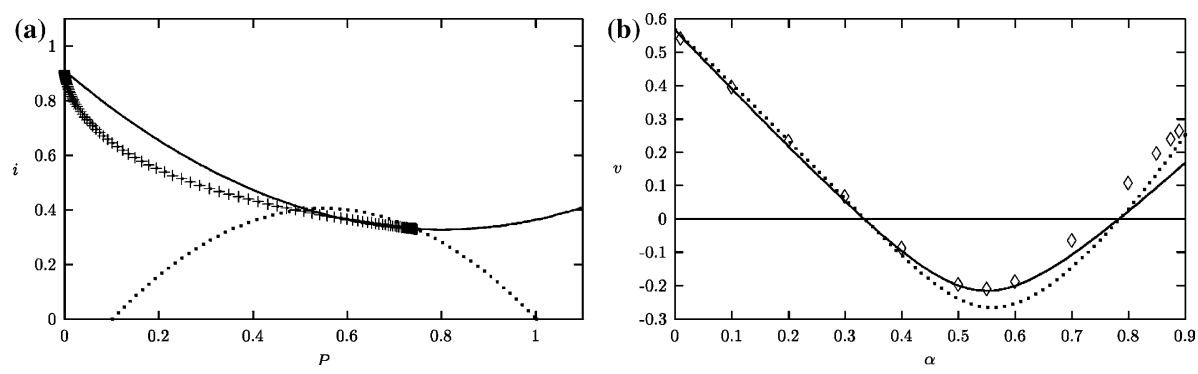

Figure 6. (a) Asymptotic front solution of the PDE system (6-9), displayed in $(P, i)$ phase space with cross points. The solid and the dotted line are the nullclines of $i$ and $P$, respectively. (b) Analytically approximated wave speeds (28) in dotted line and (30) in solid line. The points are numerically obtained results. Parameter values as in Figure 2. 

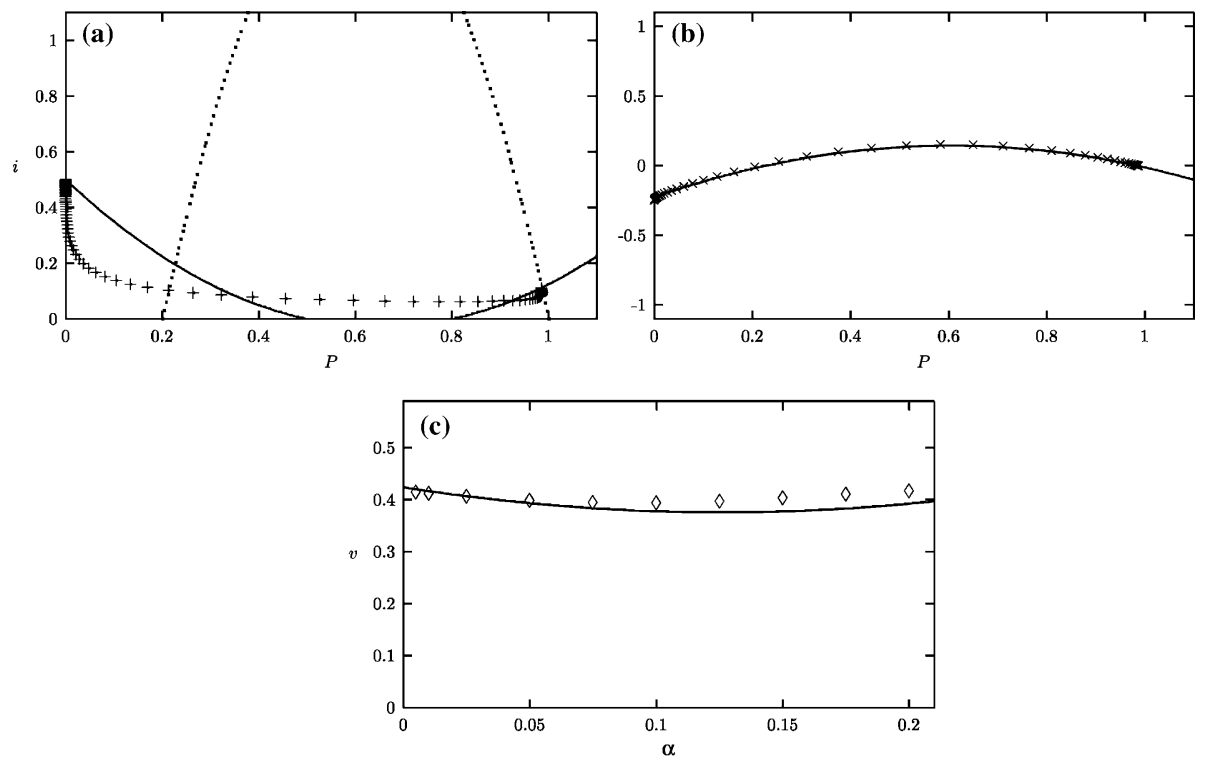

Figure 7. (a) Asymptotic front solution of the PDE system (6-9), displayed in $(P, i)$ phase plane with cross points. The solid and the dotted line are the nullcline of $i$ and $P$, respectively. (b) Approximation of the term $(1-P)(P-u)-\alpha i$ (crosses) by the right-hand side of (29) (solid line). The data for $i$ stem from the front solution of the PDE system. (c) Approximated wave speed (30) in solid line and numerically calculated data (points). Parameter values: $u=0.2, e=0.1, c=0.4, \alpha=0.1, \sigma=0.9, S_{l}=S_{3+}, I_{l}=I_{3+}, x_{S}=100$, $x_{I}=50$.

Consider for example the dynamics in Figure $6 \mathrm{a}$, which corresponds to the situation shown in Figure 2. There is still an apparent similarity between the PDE solution and the nullcline of the prevalence, cf. (16), which makes us to try to approximate $i$ in this way. I.e., we incorporate the ansatz

$$
i=1-\frac{c}{\sigma-\alpha}-\frac{1+u+e}{\sigma-\alpha} P+\frac{1}{\sigma-\alpha} P^{2},
$$

cf. (16), in Equation (21) and proceed as in subsection 4.1, thus obtaining the unique wave speed

$$
v=\sqrt{\frac{2(\sigma-\alpha)}{\sigma}}\left[\frac{3 \sigma}{2(\sigma-\alpha)} P_{3+}-1-u-\alpha \frac{1+u+e}{\sigma-\alpha}\right],
$$

with $P_{3+}$ as in (15). This analytical approximation is plotted against the virulence in Figure $6 \mathrm{~b}$ (dotted line). The predicted wave speeds closely match the numerically observed ones. Only for intermediate values of $\alpha$ are there slight underestimations. Note that the wave speed becomes positive again for large virulences, i.e. the population front propagates forward again.
There are, however, situations in which the PDE solution cannot be approximated by the nullcline, and these can naturally be assumed to be the more general case. An example is given in Figure 7a: The PDE solution would suggest rather a singular perturbation analysis than an approximation by the nullcline. Yet another approach is now set out. We seek another approximation of the term $(1-P)(P-u)-\alpha i$ in Equation (18). Let us try

$$
(1-P)(P-u)-\alpha i=\left(P_{3+}-P\right)\left(P-P_{3-}\right),
$$

of which we know that this clearly holds for $i=P_{3+}$ and $i=P_{3-}$. Note that this ansatz corresponds to the case if $i$ would be a constant (as for example in the single-species models in Murray $(2002,2003))$. Figure $7 b$ shows both the lefthand side and the right-hand side of (29) with values of $i$ from the numerical front solution. They are in good accordance for all $P \in\left[0, P_{3+}\right]$. Hence, let us make use of this approximation and substitute the term $(1-P)(P-u)-\alpha i$ in (21) by the right-hand side of (29). Then we obtain

$$
\begin{aligned}
P^{\prime} & =Q, \\
Q^{\prime} & =-v Q-\left(P_{3+}-P\right)\left(P-P_{3-}\right) P .
\end{aligned}
$$


Finally, we yield the following simple expression for the unique wave speed

$$
v=\frac{1}{\sqrt{2}}\left[P_{3+}-2 P_{3-}\right] .
$$

As we can easily see, the wave exists if the coexistence states exist. An invasion front is predicted to be reversed, if the stable coexistence state is not at least double the size of the unstable one. The spread rate is always smaller than (17), because $0<u<P_{3-}<P_{3+}<1$. This means that the introduction of the disease slows down the host population. In Figure 7c, (30) is plotted against $\alpha$ and compared with the numerically obtained wave speeds, demonstrating a good accordance. This parameter setting is an example in which the wave speeds do not become negative for feasible $\alpha$. Actually, Equation (30) can also be applied to the parameter setting in Figure 6 . The resulting wave speed approximation is plotted in Figure $6 \mathrm{~b}$ as well (solid line). Compared with (28), (30) fits very accurately for intermediate values of $\alpha$, while there is a slight underestimation for large values. However, both estimates yield the same threshold values of the virulence for the transitions from an invasive to a recessive wave and vice versa.

The wave speeds for the fast dynamics as summarized in subsection 1 can be obtained as special cases of both (28) and (30) by simply letting the respective parameters become large. All these wave speed approximations as well as the disease-free wave speed (17), are particular cases of the fundamental setting with cubic nonlinearities in the interaction term of the PDE. Say, if $r_{0}, r_{1}$, and $r_{2}$ denote the roots of this polynomial, then the wave speed is given by

$$
v=\frac{1}{\sqrt{2}}\left[r_{2}-2 r_{1}+r_{0}\right]
$$

In the case of (30) we have $r_{2}=P_{3+}, r_{1}=P_{3-}$ and $r_{0}=0$.

Finally, we are interested in the impact of the Allee threshold $u$ on the reversal dynamics. In Figure 8, the predicted wave speeds (30) are plotted against both the virulence and the Allee threshold. For fixed values of $u$, there is a minimum wave speed for some intermediate virulence $\alpha$. When the Allee threshold is varied, the wave speed decreases with $u$. This can also easily be

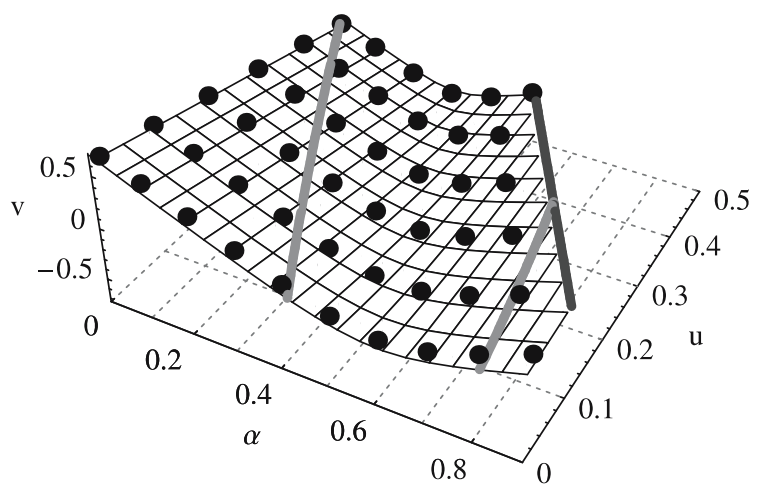

Figure 8. Dependence of the analytic wave speed solution (30) on the Allee threshold $u$ and the virulence $\alpha$. The points are numerically calculated wave speeds. The grey lines are isolines of zero wavespeed and indicate transition from a recessive to an invasive wave. The thick black line delineates the feasible parameter region in which the coexistence state exists. Other parameters chosen as in Figure 2.

checked by looking at the wave speed equations and their dependence on $u$. The parameter regions, in which a front reversal is possible, gets larger with increasing Allee threshold. Recall that for $u>0.5$ the disease-free host population wave would be recessive anyway.

\section{Discussion and conclusions}

In this paper we have considered a reactiondiffusion model describing the spatiotemporal spread of a species which is governed by a strong Allee effect as well as an infectious disease. The invasion takes place via a travelling frontal wave. It has been shown that this invasion front of the host population can be slowed down, stopped or reversed by subsequently introduced pathogens. The invasion reversal and thus the ultimate population extinction depend on the virulence. When the additional disease-related mortality is within a certain range, the deaths due to infection overbalance the growth at the head of the population front.

The asymptotic rate of propagation decreases with an increasing strength of the Allee effect, i.e. a larger threshold $u$. This agrees with the results from single-species models, in which Allee effects slow down travelling wave solutions of reactiondiffusion (Lewis and Kareiva 1993; Wang and 
Kot 2001) and integrodifference equations (Wang et al. 2002). The possible reversal of invasion fronts has also been found by Owen and Lewis (2001) in a predator-prey model where the prey exhibits an Allee effect. Predator-prey and epidemiological SI models obey some structural analogy. Critical parameters for the reversal of the fronts are the predator mortality and the diseaserelated mortality, respectively. In both the predator-prey and the infectious disease model, the local models with neglected diffusion would predict coexistence. Recessive waves ('waves of extinction') have also been observed by Lewis and van den Driessche (1993) in a model of sterile insect release, in order to control an insect invasion. It would be interesting to know, what happens in a competition or any other model with negative impact on the focal species, when the latter is subject to an Allee effect.

This study has shown that reversal is possible for Allee thresholds $u$ even approaching zero. Owen and Lewis (2001) have observed in their examples critical thresholds of about $u=0.4$ for prey-predator interactions of simple Lotka-Volterra type and of about $u=0.3$ for prey-predator interactions of Holling type III. Future work will have to consider different transmission functions. For instance, considering mass action transmission one could also investigate whether the reversal depends on the disease-induced extinction state. Since the recessive waves are possible in prey-predator systems with LotkaVolterra interaction, we would expect them also to occur in epidemiological models with transmission dynamics other than the standard incidence.

Owen and Lewis (2001) assumed that the predator diffuses much faster than the prey species, which, from a mathematical point of view, allows the application of singular perturbation theory. Here, we have considered equal diffusion coefficients of susceptibles and infected. The assumptions that some dynamical components are fast allowed the problem to be treated analytically. Alternative approaches were also proposed which are based upon numerically observed features to guide an ansatz for deriving the wave speed expression as well. The diffusion coefficient of the infected could also be assumed to be reduced as a result of the infection. Hence, the diffusion coefficients would be unequal but still of the same order of magnitude. The analysis based on the fast dynamics assumptions should also hold in this case. Whether the alternative approaches turn out to be similarly accurate, would have to be checked in respective extensive numerical simulations.

A prerequisite of the reversal, however, is that one of the species is still invading into open space, while the other one is able to catch up this front. This can be regarded to be often the case, because invasion usually takes place by introduction of a small number of individuals. When these are disease-free, the pathogen is left behind and not present in the new habitat before another introduction of infected individuals (Pimentel 1986).

Population crashes of alien invaders cannot be caused by indigenous pathogens, because they would have prevented a successful establishment. Mutational effects are more unlikely than the subsequent release of native pathogens, since evolution tends to coexistence states rather than to extinction, e.g. Roughgarden (1975), Ewald (1994), Yamamura (1996) and Boots and Sasaki (2003). Moreover, recent quantitative studies of Mitchell and Power (2003) and Torchin et al. (2003) demonstrate that invasive species, both plants and animals, appear to suffer from fewer pathogens and parasites in their new habitat than in their native range.

There are a lot of models dealing with the control of epidemic spread or investigating the impact of infectious diseases on non-native species, e.g. the control of rabies, see Murray (2003) and references therein, and immunocontraception (McCallum 1996; Courchamp and Cornell 2000; Suppo et al. 2000). Usually, the density of the host species is only reduced due to the disease, but the population does not go extinct spatially. Hence, this phenomenon can be ascribed to the Allee effect, which is another evidence that Allee dynamics are a source of complex spatiotemporal dynamics (Lewis and Kareiva 1993; Owen and Lewis 2001; Petrovskii et al. 2002a, b, 2005).

The results of this study clearly highlight the importance and role of infectious diseases in invasion processes. Furthermore, they indicate that the introduction of natural pathogens may be applicable as a biological control method, in order to eradicate a pest species or at least to slow down its spread. This depends on the availability of appropriately harmful diseases and the range of 
their release. However, possible adverse effects to the ecosystem such as transmission to indigenous species have to be taken into account, of course.

\section{Acknowledgements}

F.M.H. and H.M. gratefully acknowledge support by the Japan Society for the Promotion of Science (Predoc and Research Fellowships PE04533 and S04716, respectively), which has made possible their stays in Japan during which parts of the presented results have been obtained. F.M.H. is thankful for discussion with the groups of H. Seno, N. Shigesada, Y. Iwasa, N. Yamamura and Y. Hosono as well as with S.V. Petrovskii. M.A.L. gratefully acknowledges support from a Canada Research Chair, NSERC Collaborative Research Opportunity and Discovery grants and the National Science Foundation Grant No. DEB-0213698. The authors thank two anonymous reviewers for helpful comments.

\section{References}

Anderson PK, Cunningham AA, Patel NG, Morales FJ, Epstein PR and Daszak P (2004) Emerging infectious diseases of plants: pathogen pollution, climate change and agrotechnology drivers. Trends in Ecology \& Evolution 19(10): 535-544

Aronson DG and Weinberger HF (1975) Nonlinear diffusion in population genetics, combustion, and nerve propagation. In. Goldstein JA (ed) Partial Differential Equations and Related Topics. pp 5-49. Springer-Verlag, Berlin, Vol. 446 of Lecture Notes in Mathematics

Boots M and Sasaki A (2003) Parasite evolution and extinctions. Ecology Letters 6: 176-182

Cappuccino N (2004) Allee effect in an invasive alien plant, pale swallow-wort Vincetoxicum rossicum (Asclepiadaceae). Oikos 106: 3-8

Case TJ, Holt RD, McPeek MA and Keitt TH (2005) The community context of species' borders: ecological and evolutionary perspectives. Oikos 108: 28-46

Clark JS, Lewis M and Horvath L (2001) Invasion by extremes: population spread with variation in dispersal and reproduction. The American Naturalist 157(5): 537-554

Clay K (2003) Parasites lost. Nature 421: 585-586

Courchamp F, Clutton-Brock T and Grenfell B (1999) Inverse density dependence and the Allee effect. Trends in Ecology \& Evolution 14(10): 405-410

Courchamp F and Cornell SJ (2000) Virus-vectored immunocontraception to control feral cats on islands: a mathematical model. Journal of Applied Ecology 37: 903-913
Cruickshank I, Gurney WSC and Veitch AR (1999) The characteristics of epidemics and invasions with thresholds. Theoretical Population Biology 56: 279-292

Davis HG, Taylor CM, Civille JC and Strong DR (2004) An Allee effect at the front of a plant invasion: Spartina in a Pacific estuary. Journal of Animal Ecology 92: 321-327

de Castro F and Bolker B (2005) Mechanisms of diseaseinduced extinction. Ecology Letters 8: 117-126

Dennis B (1989) Allee effects: population growth, critical density, and the chance of extinction. Natural Resources Modelling 3: 481-538

Drake JM (2004) Allee effects and the risk of biological invasion. Risk Analysis 24(4): 795-802

Dunbar SR (1983) Travelling wave solutions of diffusive Lotka-Volterra equations. Journal of Mathematical Biology 17: $11-32$

Dunbar SR (1984) Travelling wave solutions of diffusive LotkaVolterra equations: A heteroclinic connection in $R^{4}$. Transactions of American Mathematical Society 286: 557-594

Dunbar SR (1986) Travelling waves in diffusive predator-prey equations: periodic orbits and point-to-periodic heteroclinic orbits. SIAM Journal Applied Mathematics 46: 1057-1078

Ewald PW (1994) Ecology of Infectious Diseases. Oxford University Press, Oxford

Fagan WF and Bishop JG (2000) Trophic interactions during primary succession: herbivores slow a plant reinvasion at Mount St. Helens. The American Naturalist 155: 238-251

Fagan WF, Lewis MA, Neubert MG and van den Driessche P (2002) Invasion theory and biological control. Ecology Letters 5: 148-158

Fife PC and McLeod JB (1977) The approach of solutions of nonlinear diffusion equations to travelling wave solution. Archive for Rational Mechanics and Analysis 65: 335-361

Fisher RA (1937) The wave of advance of advantageous genes. Annals of Eugenics 7: 355-369

Garrett KA and Bowden RL (2002) An Allee effect reduces the invasive potential of Tilletia indica. Phytopathology 92(11): 1152-1159

Grevstad FS (1999) Factors influencing the change of population establishment: implications of release strategies in biocontrol. Ecological Applications 9(4): 1439-1447

Gurney WSC and Nisbet RM (1998) Ecological Dynamics. Oxford University Press, New York

Gurney WSC, Veitch AR, Cruickshank I and McGeachin G (1998) Circles and spirals: population persistence in a spatially explicit predator-prey model. Ecology 79(7): 2516-2530

Hadeler KP and Rothe F (1975) Travelling fronts in nonlinear diffusion equations. Journal of Mathematical Biology 2: 251-263

Hart DR and Gardner RH (1997) A spatial model for the spread of invading organisms subject to competition. Journal of Mathematical Biology 35(8): 935-948

Hastings A, Cuddington K, Davies KF, Dugaw CJ, Elmendorf S, Freestone A, Harrison S, Holland M, Lambrinos J, Malvadkar U, Melbourne BA, Moore K, Taylor C and Thomoson D (2005) The spatial spread of invasions: new developments in theory and evidence. Ecology Letters 8: 91-101 
Hengeveld R (1989) Dynamics of Biological Invasions. Chapman and Hall, London

Hethcote HW (2000) The mathematics of infectious diseases. SIAM Review 42(4): 599-653

Hilker FM, Langlais M, Petrovskii SV and Malchow $\mathrm{H}$ (submitted) A diffusive SI model with Allee effect and application to FIV. Mathematical Biosciences

Holt RD, Keitt TH, Lewis MA, Maurer BA and Taper ML (2005) Theoretical models of species' borders: single species approaches. Oikos 108: 18-27

Hosono Y (1989) Singular perturbation analysis of travelling waves for diffusive Lotka-Volterra competitive models. In: Brezinski C (ed) Numerical and Applied Mathematics, pp 687-692. Baltzer, Basel

Huang J, Lu G and Ruan S (2003) Existence of traveling wave solutions in a diffusive predatorprey model. Journal of Mathematics Biology 46(2): 132-152

Keane RM and Crawley MJ (2002) Exotic plant invasions and the enemy release hypothesis. Trends in Ecology \& Evolution 17(4): 164-170

Keitt TH, Lewis MA and Holt RD (2001) Allee effects, invasion pinning, and species' borders. The American Naturalist 157: 203-216

Kolmogorov A, Petrovskii I and Piskunov N (1937) Étude de l'equation de la diffusion avec croissance de la quantité de matière et son application à un problème biologique. Bulletin of University Moscow, Serial Int Sec A 1: 1-25

Kot M, Lewis MA and Driessche Pvan den (1996) Dispersal data and the spread of invading organisms. Ecology 77(7): 2027-2042

Lambrinos JG (2004) How interactions between ecology and evolution influence contemporary invasion dynamics. Ecology 85(8): 2061-2070

Lee KA and Klasing KC (2004) A role for immunology in invasion biology. Trends in Ecology \& Evolution 19(10): 523-529

Leung B, Drake JM and Lodge DM (2004) Predicting invasions: propagule pressure and the gravity of Allee effects. Ecology 85(6): 1651-1660

Lewis MA (1997) Variability, patchiness, and jump dispersal in the spread of an invading population. In: Tilman D and Kareiva P (eds) Spatial Ecology. The Role of Space in Population Dynamics and Interspecific Interactions, pp 4669. Princeton University Press, Princeton, Vol 30 of Monographs in Population Biology

Lewis MA (2000) Spread rate for a nonlinear stochastic invasion. Journal of Mathematical Biology 41: 430-454

Lewis MA and Kareiva P (1993) Allee dynamics and the spread of invading organisms. Theoretical Population Biology 43: 141-158

Lewis MA and Pacala S (2000) Modeling and analysis of stochastic invasion processes. Journal of Mathematical Biology 41: 387-429

Lewis MA and van den Driessche P (1993) Waves of extinction from sterile insect release. Mathematical Biosciences 116: 221-247

Li B, Weinberger HF and Lewis MA (submitted) Existence of traveling waves for discrete and continuous time cooperative systems. Mathematical Biosciences
Liebhold A and Bascompte J (2003) The Allee effect, stochastic dynamics and the eradication of alien species. Ecology Letters 6: 133-140

Luther R (1906) Räumliche Ausbreitung chemischer Reaktionen. Zeitschrift für Elektrochemie 12: 596-600

Malchow H, Hilker FM, Petrovskii SV and Brauer K (2004) Oscillations and waves in a virally infected plankton system. I. The lysogenic stage. Ecological Complexity 1(3): 211-233

McCallum H (1996) Immunocontraception for wildlife population control. Trends in Ecology \& Evolution 11(12): 491-493

McCallum H, Barlow N and Hone J (2001) How should pathogen transmission be modelled? Trends in Ecology \& Evolution 16(6): 295-300

McKean HP (1970) Nagumo's equation. Advances in Mathematics 4: 209-223

Mitchell CE and Power AG (2003) Release of invasive plants from fungal and viral pathogens. Nature 421: 625-627

Mollison D (1991) Dependence of epidemics and populations velocities on basic parameters. Mathematical Biosciences 107: $255-287$

Murray JD (2002) Mathematical Biology. I: An Introduction. 3rd edn. Springer-Verlag, Berlin

Murray JD (2003) Mathematical Biology. II. Spatial Models and Biomedical Applications. 3rd edn. Springer-Verlag, Berlin

Nold A (1980) Heterogeneity in disease-transmission modeling. Mathematical Biosciences 52: 227-2402

Okubo A and Levin SA (2001) Diffusion and ecological problems: Modern perspectives, 2nd edn. Springer-Verlag, New York

Okubo A, Maini PK, Williamson MH and Murray JD (1989) On the spatial spread of the gray squirrel in Britain. Proceedings of the Royal Society of London B 238: 113125

Owen MR and Lewis MA (2001) How predation can slow, stop or reverse a prey invasion. Bulletin of Mathematical Biology 63: 655-684

Petrovskii SV and Malchow H (1999) A minimal model of pattern formation in a prey-predator system. Mathematical and Computer Modelling 29: 49-63

Petrovskii SV and Malchow H (2001) Wave of chaos: new mechanism of pattern formation in spatio-temporal population dynamics. Theoretical Population Biology 59(2): 157-174

Petrovskii SV, Morozov AY and Venturino E (2002a) Allee effect makes possible patchy invasion in a predator-prey system. Ecology Letters 5: 345-352

Petrovskii SV, Vinogradov ME and Morozov AY (2002b) Formation of the patchiness in the plankton horizontal distribution due to biological invasion in a two-species model with account for the Allee effect. Oceanology 42(3): 363-372

Petrovskii SV, Malchow H, Hilker FM and Venturino E (2005) Patterns of patchy spread in deterministic and stochastic models of biological invasion and biological control. Biological Invasions 7: 771-793 (this issue)

Pimentel D (1986) Biological invasions of plants and animals in agriculture and forestry. In: Mooney HA and Drake JA (eds) Ecology of Biological Invasions of North America and Hawaii, pp 149-162. Springer-Verlag, New York 
Prenter J, MacNeil C, Dick JTA and Dunn AM (2004) Roles of parasites in animal invasions. Trends in Ecology \& Evolution 19(7): 385-390

Roughgarden J (1975) Evolution of marine symbiosis - a simple cost-benefit model. Ecology 56: 1201-1208

Sherratt JA, Lewis MA and Fowler AC (1995) Ecological chaos in the wake of invasion. Proceedings of the National Academy of Sciences USA 92: 2524-2528

Sherratt JA, Eagan BT and Lewis MA (1997) Oscillations and chaos behind predator-prey invasion: mathematical artifact or ecological reality? Philosophical Transactions of the Royal Society of London B 352: 21-38

Shigesada N and Kawasaki K (1997) Biological Invasions: Theory and Practice. Oxford University Press, Oxford

Shigesada N and Kawasaki K (2002) Invasion and the range expansion of species: effects of long-distance dispersal. In: Bullock J, Kenward R and Hails R (eds) Dispersal Ecology, pp 350-373. Blackwell Science, Malden, MA

Simberloff D and Gibbons L (2004) Now you see them, now you don't! - population crashes of established introduced species. Biological Invasions 6: 161-172

Skellam JG (1951) Random dispersal in theoretical populations. Biometrika 38: 196-218

Stephens PA and Sutherland WJ (1999) Consequences of the Allee effect for behaviour, ecology and conservation. Trends in Ecology \& Evolution 14(10): 401-405

Stephens PA, Sutherland WJ and Freckleton RP (1999) What is the Allee effect? Oikos 87(1): 185-190

Suppo C, Naulin JM, Langlais M and Artois M (2000) A modelling approach to vaccination and contraception programmes for rabies control in fox populations. Proceedings of the Royal Society London B 267: 1575-1582
Takasu F, Yamamoto N, Kawasaki K, Togashi K, Kishi Y and Shigesada N (2000) Modeling the expansion of an introduced tree disease. Biological Invasions 2: 141-150

Tompkins DM, White AR and Boots M (2003) Ecological replacement of native red squirrels by invasive greys driven by disease. Ecology Letters 6: 189-196

Torchin ME, Lafferty KD and Kuris AM (2002) Parasites and marine invasions. Parasitology 124: S137-S151

Torchin ME, Lafferty KD, Dobson AP, McKenzle VJ and Kuris AM (2003) Introduced species and their missing parasites. Nature 421: 628-630

Veit RR and Lewis MA (1996) Dispersal, population growth, and the Allee effect: dynamics of the house Finch invasion of eastern North America. The American Naturalist 148(2): 255-274

Vinogradov ME, Shushkina EA, Anochina LL, Vostokov SV, Kucheruk NV and Lukashova TA (2000) Mass development of ctenophore Beroe ovata Eschscholtz off the north-eastern coast of the Black Seas. Oceanology 40: 52-55

Wang MH and Kot M (2001) Speeds of invasion in a model with strong or weak Allee effects. Mathematical Biosciences 171(1): 83-97

Wang $\mathrm{MH}$, Kot $\mathrm{M}$ and Neubert MG (2002) Integrodifference equations, Allee effects, and invasions. Journal of Mathematical Biology 44: 150-168

Williamson M. (1996) Biological Invasions. Number 15 in Population and Community Biology Series. Chapman \& Hall, London

Yamamura N (1996) Evolution of mutualistic symbiosis: a differential equation model. Researches in Population Ecology 38(2): 211-218 